UDK 61.80.Az, 74.25.Fy, 74.72.Bk, 02.70.Bf

\title{
NUMERICAL INVESTIGATION OF SCALING REGIMES IN A MODEL OF ANISOTROPICALLY ADVECTED VECTOR FIELD
}

\author{
E. Jurčišinová, ${ }^{1,2}$ M. Jurčišin, ${ }^{1,3}$ R. Remecky, ${ }^{4}$ and M. Scholtz ${ }^{4}$ \\ ${ }^{1}$ Institute of Experimental Physics, SAS, Watsonova 47, 04001 Košice, Slovakia \\ ${ }^{2}$ Laboratory of Information Technologies, JINR, 141980 Dubna, Moscow Region, Russia \\ ${ }^{3}$ Bogoliubov Laboratory of Theoretical Physics, JINR, 141980 Dubna, Moscow Region, Russia \\ ${ }^{4}$ Department of Physics and Astrophysics, Institute of Physics, P.J. Šafárik University, \\ Park Angelinum 9, 04001 Košice, Slovakia
}

\begin{abstract}
Influence of strong uniaxial small-scale anisotropy on the stability of inertial-range scaling regimes in a model of a passive transverse vector field advected by an incompressible turbulent flow is investigated by means of the field theoretic renormalization group. Turbulent fluctuations of the velocity field are taken in the form of a Gaussian statistics with zero mean and defined noise with finite correlations in time. It is shown that stability of the inertial-range scaling regimes in the three-dimensional case is not destroyed by anisotropy but the corresponding stability of the two-dimensional system can be corrupted by the presence of anisotropy. A borderline dimension $d_{c}$ below which the stability of the scaling regime is not present is calculated as a function of anisotropy parameters.
\end{abstract}

\section{INTRODUCTION}

It is well known that the breakdown of the classical Kolmogorov-Obuchov phenomenological theory of fully developed turbulence [1] is more noticeable for simpler models of passively advected scalar or vector quantity (scalar or vector field) than for the velocity field itself. This phenomenon is encoded in the terms intermittency and anomalous scaling [1, 2]. At the same time, the problem of a passive advection is easier from theoretical point of view (see, e.g., 3 and references therein). Therefore it leads to the fact that the problem of anomalous scaling can be understand here in a few ways what is not possible for the present in the problem of genuine turbulence.

One of the most effective approach for studying self-similar scaling behavior is the method of the field theoretic renormalization group (RG) 4, 5. It can be also used in the theory of fully developed turbulence and related problems [5, 6, 7], e.g., in the problem of a passive scalar (or vector) field advected by a given stochastic environment.

In [8] the field theoretic RG was applied to the so-called rapid-change model of a passive scalar advected by a self-similar white-in-time velocity field which is also known as Kraichnan model. It was shown that within the field theoretic RG approach the anomalous scaling is related to the existence of "dangerous" composite operators with negative critical dimensions in the framework of the operator product expansion (OPE) [5, 6, 7].

Afterwards, various generalized descendants of the Kraichnan model, namely, models with inclusion of large and small scale anisotropy, compressibility, and finite correlation time of the velocity field were studied by the field theoretic approach (see 9$]$ and references therein). 
Moreover, advection of a passive vector field by the Gaussian self-similar velocity field (with and without large and small scale anisotropy, pressure, compressibility, and finite correlation time) has been also investigated and all possible asymptotic scaling regimes and crossovers among them have been classified [10, 11, 12, 13. General conclusion is: the anomalous scaling, which is the most important feature of the Kraichnan rapid-change model, remains valid for all generalized models.

In what follows we shall begin with investigation of one particular model of a passive vector advected by a Gaussian velocity field with finite correlation time in the presence of the smallscale anisotropy, namely the model where the stretching term is absent (the so-called $A=0$ model, see, e.g, [1] 13]). This model is specific from several points of view but maybe the most important fact is that in contrast to the other models of passive vector admixture where the anomalous scaling is related to the composite operators built of the vector field without derivatives [12, 13] in the case under consideration it is related to the composite operators built solely of the gradients of the field. This fact radically changes the complexity of the problem especially in the anisotropic case (see, e.g., 11, 14 and references therein). Thus, in some sense, it can be consider as a further step to the nonlinear Navier-Stokes equation. In what follows, we shall present only the first part of the RG analysis, namely, we shall analyze the influence of the small-scale anisotropy on the infrared (IR) stability of the possible scaling regimes of the model. It will be seen that complexity of this task is also very close to the corresponding problem in the stochastic Navier-Stokes equation [15].

\section{FIELD THEORETIC FORMULATION OF THE MODEL}

We shall consider the model of the advection of transverse (solenoidal) passive vector field $\mathbf{b} \equiv \mathbf{b}(\mathbf{x}, t)$ which is described by the following stochastic equation

$$
\partial_{t} \mathbf{b}=\nu_{0} \Delta \mathbf{b}-(\mathbf{v} \cdot \nabla) \mathbf{b}+\mathbf{f},
$$

where $\partial_{t} \equiv \partial / \partial t, \Delta \equiv \nabla^{2}$ is the Laplace operator, $\nu_{0}$ is the diffusivity (a subscript 0 denotes bare parameters of unrenormalized theory), and $\mathbf{v} \equiv \mathbf{v}(\mathbf{x}, t)$ is incompressible advecting velocity field. The vector field $\mathbf{f} \equiv \mathbf{f}(\mathbf{x}, t)$ is a transverse Gaussian random (stirring) force with zero mean and covariance

$$
D_{i j}^{f} \equiv\left\langle f_{i}(\mathbf{x}, t) f_{j}\left(\mathbf{x}^{\prime}, t^{\prime}\right)\right\rangle=\delta\left(t-t^{\prime}\right) C_{i j}(\mathbf{r} / L), \quad \mathbf{r}=\mathbf{x}-\mathbf{x}^{\prime}
$$

where parentheses $\langle\ldots\rangle$ hereafter denote average over corresponding statistical ensemble. The noise defined in Eq. (2) maintains the steady-state of the system but the concrete form of the correlator will not be essential in what follows. The only condition which must be fulfilled by the function $C_{i j}(\mathbf{r} / L)$ is that it must decrease rapidly for $r \equiv|\mathbf{r}| \gg L$, where $L$ denotes an integral scale related to the stirring.

In real problems the velocity field $\mathbf{v}(x)$ satisfies Navier-Stokes equation but, in what follows, we shall work with a simplified model where we suppose that statistics of the velocity field is given in the form of Gaussian distribution with zero mean and pair correlation function

$$
\left\langle v_{i}(x) v_{j}\left(x^{\prime}\right)\right\rangle \equiv D_{i j}^{v}\left(x ; x^{\prime}\right)=\int \frac{d^{d} \mathbf{k} d \omega}{(2 \pi)^{d+1}} R_{i j}(\mathbf{k}) D^{v}(\omega, \mathbf{k}) e^{-i \omega\left(t-t^{\prime}\right)+i \mathbf{k}\left(\mathbf{x}-\mathbf{x}^{\prime}\right)}
$$


where $d$ is the dimension of the space, $\mathbf{k}$ is the wave vector, and $R_{i j}(\mathbf{k})$ is a transverse projector. In our uniaxial anisotropic case it is taken as (see, e.g., [12] and references therein)

$$
R_{i j}(\mathbf{k})=\left(1+\alpha_{1}(\mathbf{n} \cdot \mathbf{k})^{2} / k^{2}\right) P_{i j}(\mathbf{k})+\alpha_{2} n_{s} n_{l} P_{i s}(\mathbf{k}) P_{j l}(\mathbf{k}),
$$

where $P_{i j}(\mathbf{k}) \equiv \delta_{i j}-k_{i} k_{j} / k^{2}$ is common isotropic transverse projector, the unit vector $\mathbf{n}$ determines the distinguished direction, and $\alpha_{1}, \alpha_{2}$ are parameters characterizing the anisotropy. From the positiveness of the correlation tensor $D_{i j}^{v}$ one immediately finds restrictions on the values of the above parameters, namely $\alpha_{1,2}>-1$. The function $D^{v}(\omega, \mathbf{k})$ in (3) is taken in the following form 13 .

$$
D^{v}(\omega, k)=\frac{g_{0} u_{0} \nu_{0}^{3} k^{4-d-2 \varepsilon-\eta}}{\left(i \omega+u_{0} \nu_{0} k^{2-\eta}\right)\left(-i \omega+u_{0} \nu_{0} k^{2-\eta}\right)},
$$

where $g_{0}$ plays the role of the coupling constant of the model (a formal small parameter of the ordinary perturbation theory), the parameter $u_{0}$ gives the ratio of turnover time of scalar field and velocity correlation time, and the positive exponents $\varepsilon$ and $\eta$ are small RG expansion parameters. The coupling constant $g_{0}$ and the exponent $\varepsilon$ control the behavior of the equal-time pair correlation function of velocity field and the parameter $u_{0}$ together with the second exponent $\eta$ are related to the frequency $\omega \simeq u_{0} \nu_{0} k^{2-\eta}$ which characterizes the mode $k$. The value $\varepsilon=4 / 3$ corresponds to the celebrated Kolmogorov "two-thirds law" for the spatial statistics of velocity field, and $\eta=4 / 3$ corresponds to the Kolmogorov frequency. Simple dimensional analysis shows that $g_{0}$ and $u_{0}$, which we commonly term as charges, are related to the characteristic ultraviolet (UV) momentum scale $\Lambda$ (or inner legth $l \sim \Lambda^{-1}$ ) by

$$
g_{0} \simeq \Lambda^{2 \varepsilon}, \quad u_{0} \simeq \Lambda^{\eta} .
$$

The stochastic problem (11)-(3) can be treated as a field theory with action functional [4, 5]

$$
\begin{aligned}
S(\Phi)= & b_{j}^{\prime}\left[\left(-\partial_{t}-v_{i} \partial_{i}+\nu_{0} \Delta+\nu_{0} \chi_{10}(\mathbf{n} \cdot \partial)^{2}\right) \delta_{j k}+n_{j} \nu_{0}\left(\chi_{20} \Delta+\chi_{30}(\mathbf{n} \cdot \partial)^{2}\right) n_{k}\right] b_{k} \\
& -\frac{1}{2}\left(v_{i}\left[D_{i j}^{v}\right]^{-1} v_{j}-b_{i}^{\prime} D_{i j}^{f} b_{j}^{\prime}\right),
\end{aligned}
$$

where $D_{i j}^{v}$ and $D_{i j}^{f}$ are given in (3) and (2) respectively, $\mathbf{b}^{\prime}$ is an auxiliary vector field (see, e.g., [5]), and the required integrations over $x=(\mathbf{x}, t)$ and summations over the vector indices are implied. In action (7) the terms with new parameters $\chi_{10}, \chi_{20}$, and $\chi_{30}$ are related to the presence of small-scale anisotropy and they are necessary to make the model multiplicatively renormalizable. Model (7) corresponds to a standard Feynman diagrammatic technique (see, e.g., 12, 15] for details) and the standard analysis of canonical dimensions then shows which one-irreducible Green functions can possess UV superficial divergences.

The functional formulation (77) gives possibility to use the field-theoretic methods, including the RG technique to solve the problem. By means of the RG approach it is possible to extract large-scale asymptotic behavior of the correlation functions after an appropriate renormalization procedure which is needed to remove UV-divergences. 


\section{SCALING REGIMES OF THE MODEL}

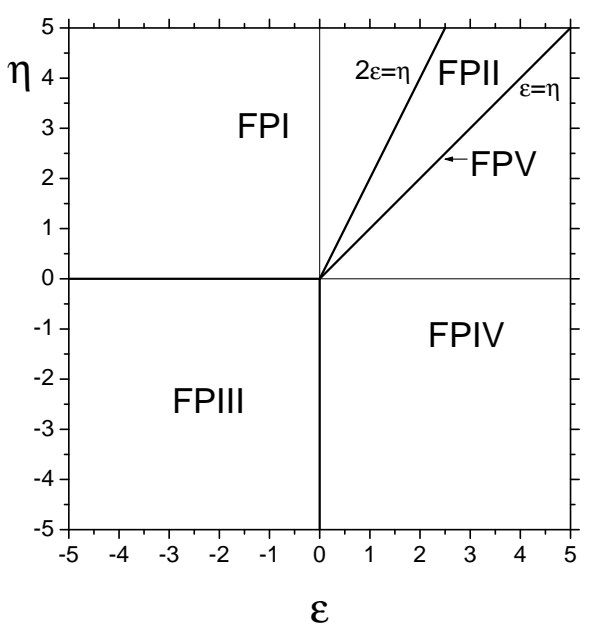

Fig. 1: The scaling regimes of the model in the $\varepsilon-\eta$ plane. The region FPI corresponds to the trivial rapid-change limit: $g^{*} / u^{*}=0,1 / u^{*}=0$. The region FPII corresponds to the the non-trivial rapid change limit: $g^{*} / u^{*}>0,1 / u^{*}=0$. The region FPIII corresponds the trivial "frozen" limit: $g^{*}=0, u^{*}=0$. The region FPIV is related to the non-trivial "frozen" limit: $g^{*}>0, u^{*}=0$. In the end, the line $\varepsilon=\eta$ (FPV) corresponds to the more interesting scaling regime with $g^{*}>0,0<u^{*}<\infty$.

Details of the one-loop field-theoretic RG analysis of the model will be done elsewhere. Here we only conclude that using the RG analysis leads to the following result: possible scaling regimes are given by the IR stable fixed points of the system of five nonlinear RG differential equations (flow equations or also known as Gell-Mann-Low equations) for five scale dependent effective variables (charges) $\bar{C}=\left\{\bar{g}, \bar{u}, \bar{\chi}_{1}, \bar{\chi}_{2}, \bar{\chi}_{3}\right\}$ of the model which are functions of the dimensionless scale parameter $t=k / \Lambda$ [5] [15]. The system of the flow equations is defined by the so-called $\beta$-functions of the model (they are functions of the charges, anisotropy parameters, space dimension, and parameters $\varepsilon, \eta)$ and it has the following form

$$
\begin{aligned}
t \frac{d \bar{g}}{d t}= & \beta_{g}=\bar{g}\left(-2 \varepsilon+2 \gamma_{1}\right), \\
t \frac{d \bar{u}}{d t}= & \beta_{u}=\bar{u}\left(-\eta+\gamma_{1}\right), \\
t \frac{d \bar{\chi}_{i}}{d t}= & \beta_{\chi_{i}}=\bar{\chi}_{i}\left(\gamma_{1}-\gamma_{i+1}\right), \\
& i=1,2,3,
\end{aligned}
$$

where the functions $\gamma_{i}, i=1,2,3,4$ are given by the following expressions (the one-loop approximation)

$$
\begin{aligned}
\gamma_{1} & =-g \frac{S_{d-1}}{(2 \pi)^{d}} \frac{1}{2(d-1)(d+1)} \int_{-1}^{1} d x \frac{\left(1-x^{2}\right)^{(d-3) / 2}}{w_{1} w_{2}} K_{1}, \\
\gamma_{i+1} & =-\frac{g}{\chi_{i}} \frac{S_{d-1}}{(2 \pi)^{d}} \frac{1}{2(d-1)(d+1)} \int_{-1}^{1} d x \frac{\left(1-x^{2}\right)^{(d-3) / 2}}{w_{1} w_{2}} K_{i+1}, \quad i=1,2,3
\end{aligned}
$$

where $S_{d}=2 \pi^{d / 2} / \Gamma(d / 2)$ is the surface of the $d$ dimensional sphere, $w_{1}=\left(1+u+\chi_{1} x^{2}\right), w_{2}=$ $\left(1+u+\chi_{1} x^{2}+\left(\chi_{2}+\chi_{3} x^{2}\right)\left(1-x^{2}\right)\right)$, and the coefficients $K_{i}\left(x^{2}, g, u, \chi_{1}, \chi_{2}, \chi_{3}, \alpha_{1}, \alpha_{2}, d\right), i=$ $1,2,3,4$ are some huge polynomials in respect to all variables and their explicit form will be given elsewhere. The scale parameter $t$ belongs to the interval $0 \leq t \leq 1$ with the initial conditions given at $t=1$ and the IR stable fixed point corresponds to the limit $t \rightarrow 0$, i.e., $\left.\bar{C}\right|_{t=0}=C^{*}$.

We have performed a numerical analysis of this system of differential equations and our aim was twofold. First of all, we have found all possible scaling regimes and we have analyzed the regions of their IR stability in the $\varepsilon-\eta$ plane. The results of this analysis are shown in Fig.1] where it is shown that the model exhibits five different scaling regimes (two for rapid-change 

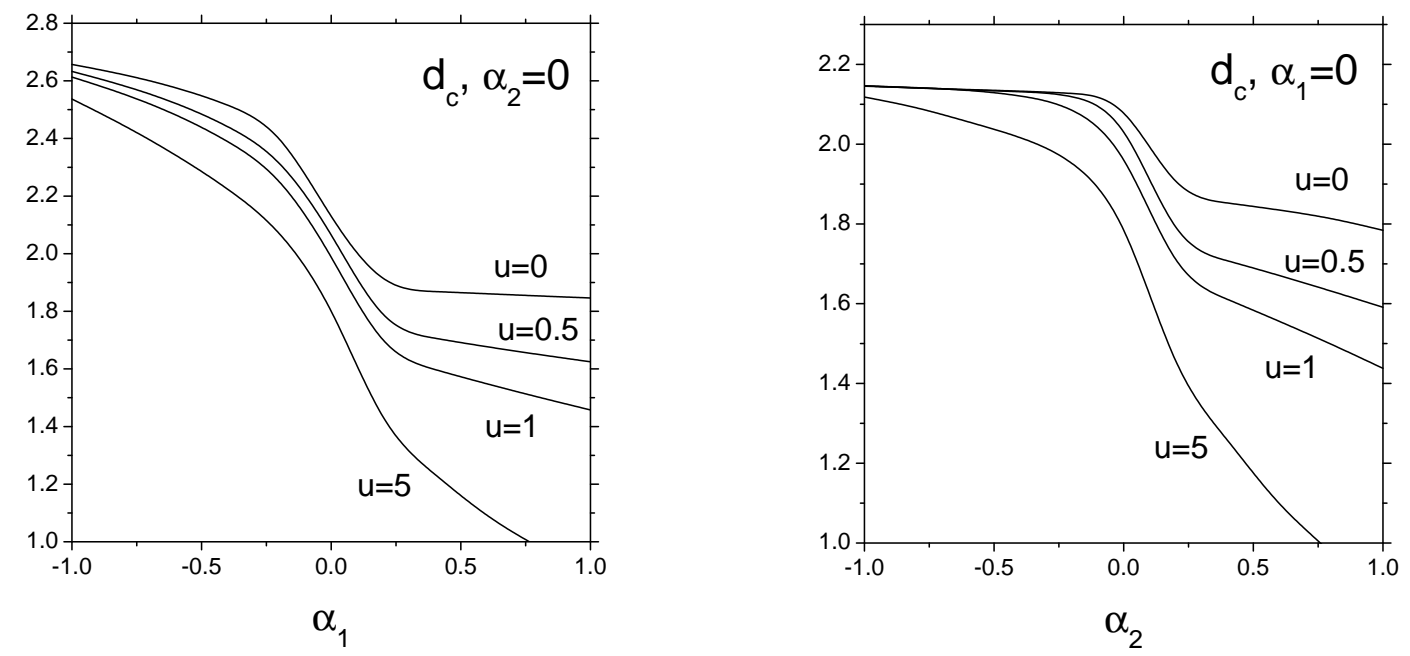

Fig. 2: Dependence of the borderline dimension $d_{c}$ on the parameters $\alpha_{1}$ and $\alpha_{2}$ for different values of $u=u^{*}$. The corresponding scaling regime is stable above the given curve.

limit, two for so-called "frozen" limit, and one general with nonzero $u_{*}$ ) (see, e.g., 13 and references therein). The second question which was investigated is related to the dependence of stability of the above mentioned scaling regimes on the anisotropy parameters $\alpha_{1}, \alpha_{2}$ and on the dimension of the space $d$. We have found the so-called borderline dimension $d_{c}$ between stable and unstable regimes as a function of anisotropy parameters $\alpha_{1}, \alpha_{2}$ and parameter $u_{*}$. The results are shown in Fig.2 for some special situations. One can see that the presence of small-scale anisotropy leads to the violation of the stability of the corresponding scaling regimes below $d_{c} \in[2,3]$ for appropriate values of anisotropy parameters. But from the point of view of further investigation of anomalous scaling the most important conclusion is that all the three-dimensional scaling regimes remain stable under influence of small-scale uniaxial anisotropy.

\section{CONCLUSIONS}

Using the field theoretic RG we have studied the influence of small-scale uniaxial anisotropy on the stability of the scaling regimes in the model of a passive vector advected by given stochastic environment with finite time correlations. It is shown that the system exhibits five different scaling regimes. They are related to the values of the parameters $\varepsilon$ and $\eta$. On the other hand, the stability of all these scaling regimes are influenced by presence of small-scale anisotropy which is demonstrated in the existence of the so-called borderline dimension $d_{c}$ which is a function of the anisotropy parameters. The $d_{c}$ is defined as dimension above which the corresponding scaling regime is stable and below which the stability of the regime is destroyed. All calculations have been done at the one-loop level. The results will be used in the further investigations of the anomalous scaling of the model.

E.J. is thankful to J. Buša for discussion. The work was supported in part by VEGA grant 
6193 of Slovak Academy of Sciences and by Science and Technology Assistance Agency under contract No. APVT-51-027904.

\section{REFERENCES}

[1] U. Frisch, Turbulence: The Legacy of A.N. Kolmogorov (Cambridge University Press, Cambridge, 1995).

[2] A. S. Monin, A. M Yaglom, Statistical Fluid Mechanics (MIT Press, Cambridge, MA, 1975), Vol. 2.

[3] G. Falkovich, K. Gawędzki, M. Vergassola, Rev. Mod. Phys. 73, 913 (2001).

[4] J. Zinn-Justin, Quantum Field Theory and Critical Phenomena (Clarendon, Oxford, 1989).

[5] A. N. Vasil'ev, Quantum-Field Renormalization Group in the Theory of Critical Phenomena and Stochastic Dynamics (St. Petersburg Institute of Nuclear Physics, St. Petersburg, 1998) [in Russian; English translation: Gordon \& Breach, 2004].

[6] L. Ts. Adzhemyan, N. V. Antonov, and A. N. Vasil'ev, Usp. Fiz. Nauk 166, 1257 (1996) [Phys. Usp. 39, 1193 (1996)].

[7] L. Ts. Adzhemyan, N. V. Antonov, and A. N. Vasil'ev, The Field Theoretic Renormalization Group in Fully Developed Turbulence (Gordon \& Breach, London, 1999).

[8] L. Ts. Adzhemyan, N. V. Antonov, and A. N. Vasil'ev, Phys. Rev. E 58, 1823 (1998).

[9] N.V. Antonov, J. Phys. A: Math. Gen. 39, 7825 (2006).

[10] N. V. Antonov, A. Lanotte, and A. Mazzino, Phys. Rev. E 61, 6586 (2000); N. V. Antonov, J. Honkonen, A. Mazzino, and P. Muratore-Ginanneschi, ibid. 62, R5891 (2000).

[11] L. Ts. Adzhemyan, N. V. Antonov, and A. V. Runov, Phys. Rev. E 64, 046310 (2001).

[12] M. Hnatic, M. Jurcisin, A. Mazzino, S. Sprinc, acta phys. slov. 52, 559 (2002); M. Hnatic, J. Honkonen, M. Jurcisin, A. Mazzino, and S. Sprinc, Phys. Rev. E 71, 066312 (2005).

[13] N.V. Antonov, M. Hnatic, J. Honkonen, and M. Jurcisin, Phys. Rev. E 68, 046306 (2003);

[14] S.V. Novikov, J. Phys. A: Math. Gen. 39, 8133 (2006).

[15] J. Buša, M. Hnatich, J. Honkonen, and D. Horvath, Phys. Rev. E 55381 (1997); J. Buša, M. Hnatich, E. Jurčišinová, M. Jurčišin, M. Stehlik, acta phys. slov. 52, 547 (2002); E.A. Hayryan, E. Jurcisinova, M. Jurcisin, I. Pokorny, M. Stehlik, Communication of JINR E17-2005-208. 